\title{
Identification of microRNAs as potential biomarkers for lung adenocarcinoma using integrating genomics analysis
}

\author{
Zhuo Peng ${ }^{1}$, Longfei Pan ${ }^{1}$, Zequn Niư ${ }^{1}$, Wei Li ${ }^{2}$, Xiaoyan Dang ${ }^{1}$, Lin Wan ${ }^{1}$, Rui \\ Zhang ${ }^{1}$ and Shuanying Yang ${ }^{2}$ \\ ${ }^{1}$ Department of Emergency Medicine, Second Affiliated Hospital, Xi'an Jiaotong University, Xi'an 710004, Shaanxi Province, \\ China \\ ${ }^{2}$ Department of Respiratory Medicine, Second Affiliated Hospital, Xi'an Jiaotong University, Xi'an 710004, Shaanxi Province, \\ China \\ Correspondence to: Shuanying Yang, email: yangshuanying66@163.com \\ Keywords: microRNAs, lung adenocarcinoma, robust rank aggregation \\ Received: March 20, $2017 \quad$ Accepted: June 05, $2017 \quad$ Published: July 18, 2017 \\ Copyright: Peng et al. This is an open-access article distributed under the terms of the Creative Commons Attribution License 3.0 \\ (CC BY 3.0), which permits unrestricted use, distribution, and reproduction in any medium, provided the original author and source \\ are credited.
}

\section{ABSTRACT}

Lung adenocarcinoma (LUAD) is the most common histological subtype of nonsmall cell lung cancer, but novel biomarkers for early diagnosis are lacking. Extensive effort has been exerted to identify miRNA biomarkers in LUAD. Unfortunately, high inter-lab variability and small sample sizes have produced inconsistent conclusions in this field. To resolve the above-mentioned limitations, we performed a comprehensive analysis based on LUAD miRNome profiling studies using the robust rank aggregation (RRA) method. Moreover, miRNA-gene interaction network, pathway enrichment analysis and Kaplan-Meier survival curves were used to investigate the clinical values and biological functions of the identified miRNAs. A total of six common differentially expressed miRNAs (DEMs) were identified in LUAD. An independent cohort further confirmed that four miRNAs (miR-21-5p, miR-210-3p, miR-182-5p and miR-183-5p) were up-regulated and two miRNAs (miR-126-3p and miR-218-5p) were down-regulated in LUAD tissues. Pathway enrichment analysis also suggested that the above-listed six DEMs may affect LUAD progression via the estrogen signaling pathway. Survival analysis based on the TCGA dataset revealed the potential prognostic values of six DEMs in patients with LUAD ( $P$-value $<0.01)$. In conclusion, we identified a panel of six miRNAs from LUAD using miRNome profiling studies. Our results provide evidence for the use of these six DEMs as novel diagnostic and prognostic biomarkers for LUAD patients.

\section{INTRODUCTION}

The incidence and mortality of lung cancer have increased steadily worldwide in the past several decades. Lung cancer is a fatal disease that is tightly associated with cigarette smoking. Apart from other histological subtypes, lung adenocarcinoma (LUAD) is the most common histological subtype among never-smokers [1]. Despite many achievements made in anti-cancer therapy over years, the survival of LUAD is far from satisfactory. Due to a lack of specific clinical symptoms, most patients with LUAD are diagnosed at a late stage, leaving little chance for effective treatment. Thus, exploring novel cancer-specific biomarkers for LUAD patients will help monitor tumor progression and guide clinical treatment.

MiRNAs are a group of small molecules that facilitate tumor progression via regulation of the function of protein-coding mRNAs [2]. MiRNAs are stable molecules that exist in tissue and fluid samples and exhibit potential as biomarkers for diagnostic, prognostic and therapeutic applications in multiple malignancies [3]. The application of high-throughput miRNA profiling methods during the past decade, such as RNA sequencing and microarrays, has enabled researchers to identify a group of miRNAs 
as biomarkers in cancer diagnosis [4]. The majority of previous studies were primarily based on small specimen sizes and various technological platforms, and no consistent conclusion has ever been made. To overcome these limitations, Vosa et al developed a robust rank aggregation (RRA) method for comparing several ranked gene lists and identifying the most commonly overlapping miRNAs [5]. By applying the RRA method, Vosa et al identified 15 aberrantly expressed miRNAs in lung cancer [6]. However, there has been no attempt to investigate LUAD-specific miRNAs using the RRA method.

In the present study, we first performed RRA analysis based on high-throughput miRNome profiling studies and identified candidate miRNAs in LUAD tissues. We next validated the aberrant miRNAs identified in the first step in an independent cohort. Finally, we predicted the biological functions of the above-identified DEMs and investigated the clinical values of these miRNAs in The Cancer Genome Atlas (TCGA) cohort.

\section{RESULTS}

\section{Characteristics of included miRNome profiling studies}

A total of nine miRNome profiling studies retrieved from the PubMed database (https://www.ncbi. nlm. nih.gov/pubmed/) were used to construct the lung adenocarcinoma miRNA expression profiling datasets [715]. Supplementary Table 1 lists the basic characteristics of the included nine miRNome profiling studies, including the first author, publication year, first author initials, country, population ethnicity, sample number (tumor tissues and normal/benign tissues), sample source (frozen or formalin-fixed paraffin-embedded tissues), detection methods, detected miRNA number, and cut-off criteria for miRNA selection. The pooled dataset included 595 cancer and 168 non-cancerous tissue samples. Various microarray platforms were used in the studies, such as

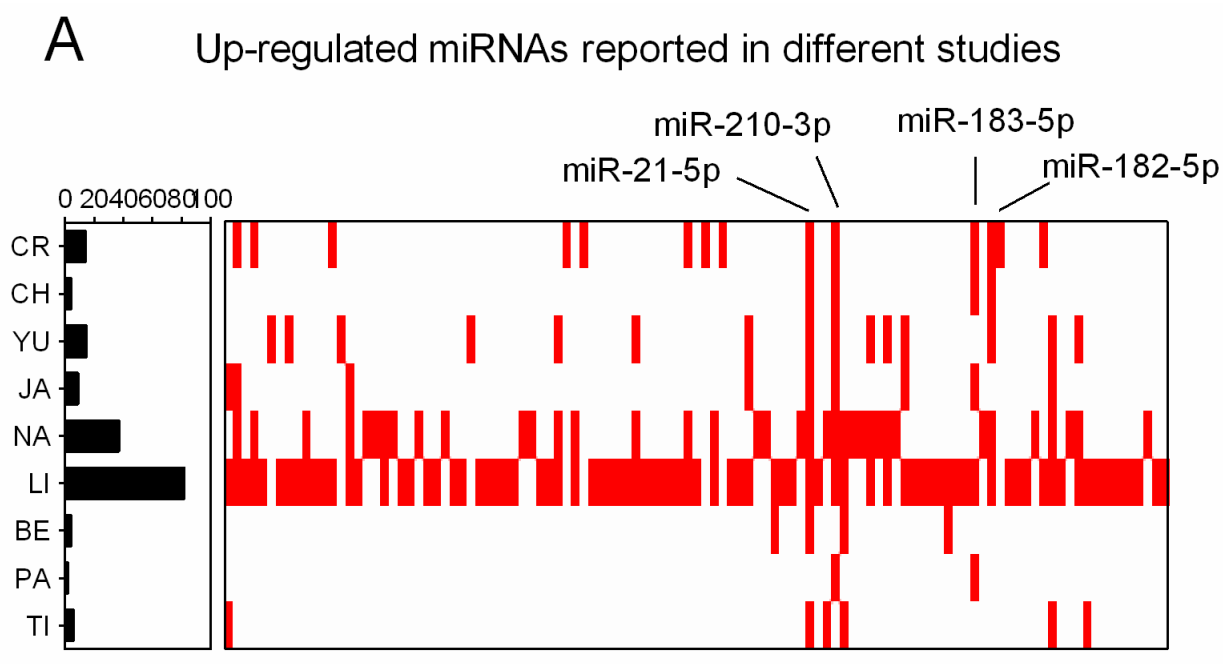

B Down-regulated miRNAs reported in different studies

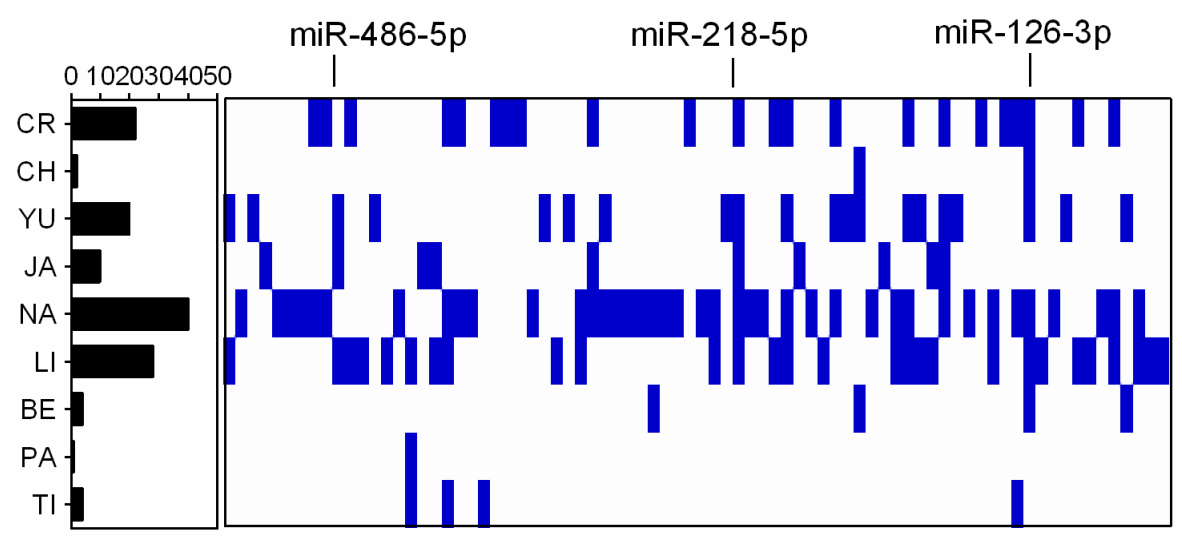

Figure 1: Distribution of DEMs in miRNome profiling studies. (A) Up-regulated miRNAs reported in nine LUAD miRNome profiling studies. (B) Down-regulated miRNAs reported in nine LUAD miRNome profiling studies. The positions of DEMs were assigned in the figure. 
Table 1: Differentially expressed miRNAs in lung adenocarcinoma

\begin{tabular}{|c|c|c|c|}
\hline microRNA & Chromosome & $P$-value & Corrected $\boldsymbol{P}$-value \\
\hline \multicolumn{4}{|l|}{ Up-regulated } \\
\hline $\operatorname{miR}-21-5 p$ & $17 \mathrm{q} 23.1$ & $1.09 \mathrm{E}-09$ & $1.14 \mathrm{E}-06$ \\
\hline $\operatorname{miR}-210-3 p$ & $11 \mathrm{p} 15.5$ & 1.35E-09 & $1.41 \mathrm{E}-06$ \\
\hline miR-182-5p & $7 \mathrm{q} 32.2$ & $1.38 \mathrm{E}-05$ & $1.44 \mathrm{E}-02$ \\
\hline miR-183-5p & $7 q 32.2$ & $1.42 \mathrm{E}-05$ & $1.49 \mathrm{E}-02$ \\
\hline miR-9-5p & $1 \mathrm{q} 22$ & 4.84E-05 & $5.07 \mathrm{E}-02$ \\
\hline miR-135b-5p & $1 \mathrm{q} 32.1$ & 4.92E-05 & $5.15 \mathrm{E}-02$ \\
\hline $\operatorname{miR}-9-3 p$ & $5 q 14.3$ & $1.22 \mathrm{E}-03$ & $1.00 \mathrm{E}+00$ \\
\hline $\operatorname{miR}-96-5 p$ & $7 q 32.2$ & $4.85 \mathrm{E}-03$ & $1.00 \mathrm{E}+00$ \\
\hline $\operatorname{miR}-205-5 p$ & $1 \mathrm{q} 32.2$ & 5.39E-03 & $1.00 \mathrm{E}+00$ \\
\hline $\operatorname{miR}-31-5 p$ & $9 \mathrm{p} 21.3$ & $6.56 \mathrm{E}-03$ & $1.00 \mathrm{E}+00$ \\
\hline miR-708-5p & $11 \mathrm{q} 14.1$ & $1.88 \mathrm{E}-02$ & $1.00 \mathrm{E}+00$ \\
\hline miR-196b-5p & $7 \mathrm{p} 15.2$ & $2.11 \mathrm{E}-02$ & $1.00 \mathrm{E}+00$ \\
\hline $\operatorname{miR}-375$ & $2 q 35$ & $2.43 \mathrm{E}-02$ & $1.00 \mathrm{E}+00$ \\
\hline miR-345-5p & $14 \mathrm{q} 32.2$ & $2.64 \mathrm{E}-02$ & $1.00 \mathrm{E}+00$ \\
\hline miR-200a-3p & $1 \mathrm{p} 36.33$ & 3.07E-02 & $1.00 \mathrm{E}+00$ \\
\hline $\operatorname{miR}-130 b-3 p$ & $22 \mathrm{q} 11.21$ & $3.31 \mathrm{E}-02$ & $1.00 \mathrm{E}+00$ \\
\hline \multicolumn{4}{|l|}{ Down-regulated } \\
\hline miR-126-3p & $9 \mathrm{q} 34.3$ & $1.34 \mathrm{E}-08$ & $1.40 \mathrm{E}-05$ \\
\hline miR-218-5p & $4 \mathrm{p} 15.31$ & 8.33E-06 & 8.71E-03 \\
\hline $\operatorname{miR}-486-5 p$ & $8 \mathrm{p} 11.21$ & 4.16E-05 & 4.35E-02 \\
\hline $\operatorname{miR}-145-5 p$ & $5 q 32$ & $1.10 \mathrm{E}-04$ & $1.15 \mathrm{E}-01$ \\
\hline miR-338-3p & $17 \mathrm{q} 25.3$ & 2.87E-04 & $3.00 \mathrm{E}-01$ \\
\hline miR-195-5p & $17 \mathrm{p} 13.1$ & $5.04 \mathrm{E}-04$ & $5.28 \mathrm{E}-01$ \\
\hline miR-143-3p & $5 q 32$ & $8.14 \mathrm{E}-04$ & $8.51 \mathrm{E}-01$ \\
\hline miR-139-5p & $11 \mathrm{q} 13.4$ & $1.66 \mathrm{E}-03$ & $1.00 \mathrm{E}+00$ \\
\hline miR-126-5p & $9 q 34.3$ & $1.66 \mathrm{E}-03$ & $1.00 \mathrm{E}+00$ \\
\hline miR-144-3p & $17 \mathrm{q} 11.2$ & 2.63E-03 & $1.00 \mathrm{E}+00$ \\
\hline miR-34c-5p & $11 \mathrm{q} 23.1$ & $2.69 \mathrm{E}-03$ & $1.00 \mathrm{E}+00$ \\
\hline miR-30a-3p & $6 q 13$ & $2.88 \mathrm{E}-03$ & $1.00 \mathrm{E}+00$ \\
\hline let- $7 c-5 p$ & $21 \mathrm{q} 21.1$ & $1.65 \mathrm{E}-02$ & $1.00 \mathrm{E}+00$ \\
\hline miR-451a & $17 \mathrm{q} 11.2$ & $2.00 \mathrm{E}-02$ & $1.00 \mathrm{E}+00$ \\
\hline miR-1-3p & $18 \mathrm{q} 11.2$ & 3.39E-02 & $1.00 \mathrm{E}+00$ \\
\hline miR-133a-3p & $20 \mathrm{q} 13.33$ & $3.47 \mathrm{E}-02$ & $1.00 \mathrm{E}+00$ \\
\hline
\end{tabular}

high-throughput quantitative PCR (qPCR) and microarraybased and second-generation sequencing methods. The numbers of detected miRNAs in each study varied from 84 to 1046.

\section{Differentially expressed miRNAs (DEMs) in LUAD}

Figure 1 shows the 109 up-regulated and 78 downregulated miRNAs that were reported in at least one 
study. Eighteen up-regulated $(16.5 \%, 18 / 109)$ and 13 down-regulated $(16.7 \%, 13 / 78)$ miRNAs were reported in more than three studies. As previously reported [16], we performed an integrated analysis using the $\mathrm{R}$ package "Robust Rank Aggreg". We primarily identified a statistically significant panel of 16 up-regulated miRNAs (miR-21-5p, miR-210-3p, miR-182-5p, miR-183-5p, miR9-5p, miR-135b-5p, miR-9-3p, miR-96-5p, miR-205-5p, miR-31-5p, miR-708-5p, miR-196b-5p, miR-375, miR345-5p, miR-200a-3p, and miR-130b-3p) and 16 down-

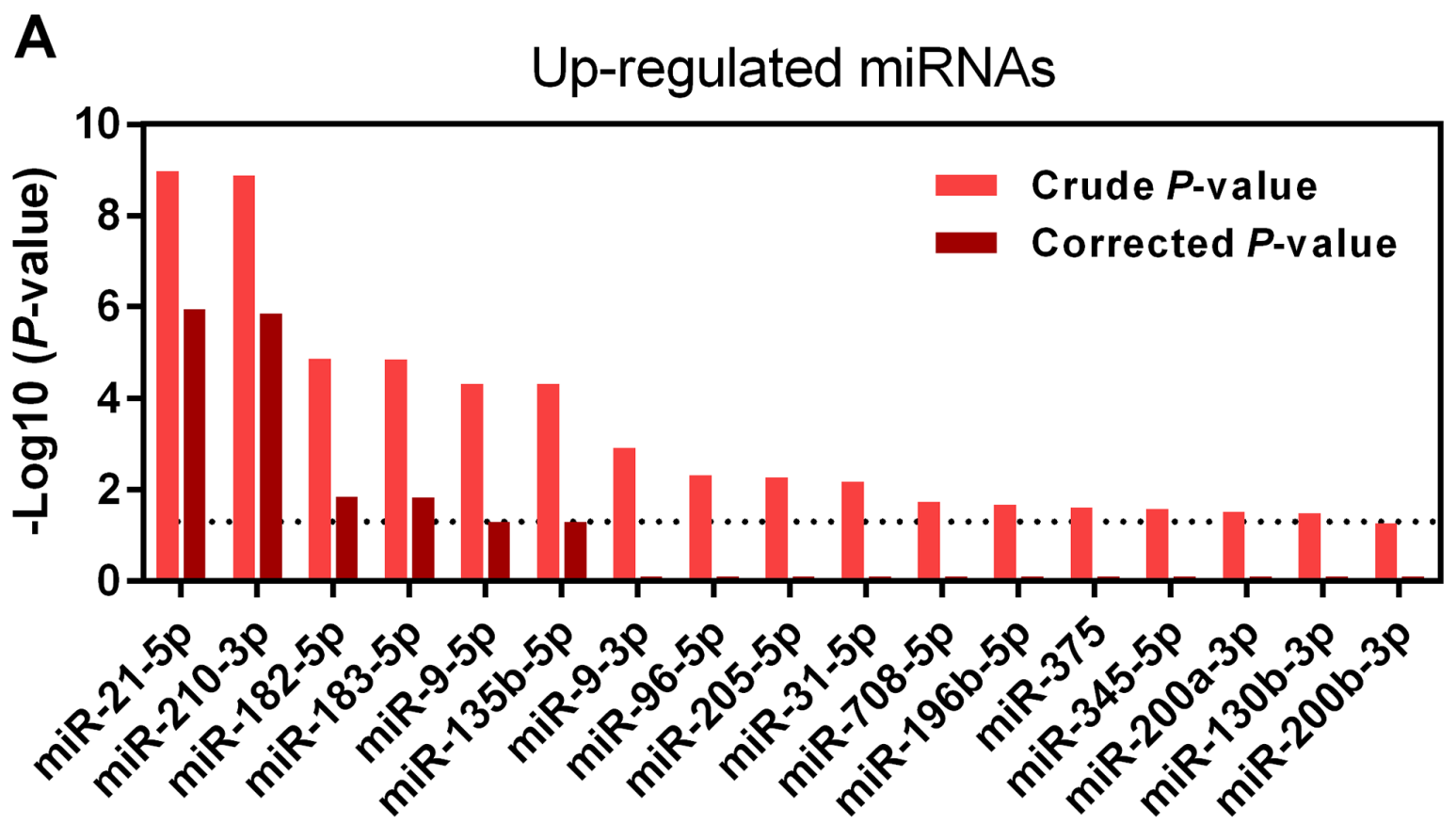

B Down-regulated miRNAs

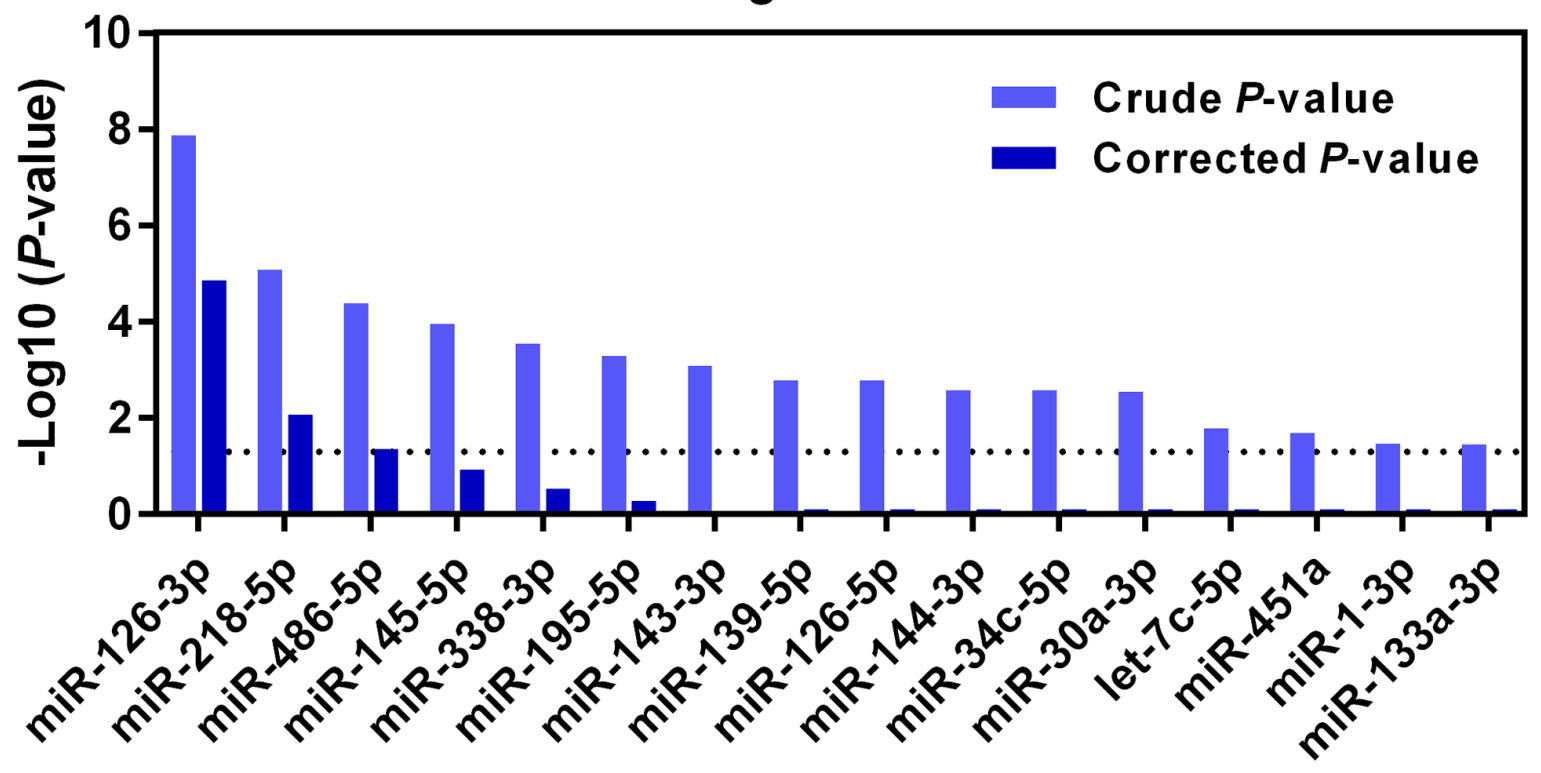

Figure 2: Ranks of six DEMs in miRNome profiling studies. (A) The ranks of four up-regulated miRNAs (miR-21-5p, miR-2103p, miR-182-5p and miR-183-5p) in nine LUAD miRNome profiling studies. (B) The ranks of two down-regulated miRNAs (miR-126-3p and miR-218-5p) in nine LUAD miRNome profiling studies. 
regulated miRNAs (miR-126-3p, miR-218-5p, miR-4865p, miR-145-5p, miR-338-3p, miR-195-5p, miR-143-3p, miR-139-5p, miR-126-5p, miR-144-3p, miR-34c-5p, miR-30a-3p, let-7c-5p, miR-451a, miR-1-3p, and miR133a-3p) (Table 1). The direction of expression change of these miRNAs was consistent across all miRome profiling studies, with $P$-values ranging from $3.47 \mathrm{E}-02$ to $1.09 \mathrm{E}-09$. Four of the up-regulated miRNAs (miR-21-5p, miR-210$3 p$, miR-182-5p and miR-183-5p) and two down-regulated miRNAs (miR-126-3p and miR-218-5p) remained statistically significant after Bonferroni correction (corrected $P$-value from 1.49E-02 to 1.14E-06) (Table 1 and Figure 2). These six miRNAs exhibited relatively high rank scores across the included studies, which supports their potential clinical values as biomarkers for LUAD (Figure 3).

\section{Validation of DEMs in LUAD tissues}

To further validate the expression levels of six DEMs in LUAD patients, we downloaded an independent cohort (GSE51853) from the GEO database (https:// www.ncbi.nlm.nih.gov/geo/), which contained five different histological types of lung cancer, including adenocarcinoma $(n=76)$, adenosquamous carcinoma $(n=4)$, large cell carcinoma $(n=15)$, large cell neuroendocrine carcinoma $(n=2)$ and squamous cell carcinoma $(n=29)$. In this microarray dataset, normal lung tissues were used as controls in this microarray dataset. MiRNA expression profiles in LUAD patients revealed that miR-21-5p, miR- 210-3p, miR-182-5p and miR-183-5p were overexpressed in nearly all samples. In contrast, miR-126-3p and miR218-5p were significantly under-expressed (Figure 4A). Comparison of miRNA expression patterns between LUAD and normal lung tissues also revealed that miR21-5p, miR-210-3p, miR-182-5p and miR-183-5p were significantly up-regulated and miR-126-3p and miR-218$5 \mathrm{p}$ were down-regulated in LUAD tissues (all $P<0.01$, Figure 4B).

\section{Functional prediction of DEMs}

The main function of miRNA is the silencing of gene expression via binding to specific target sites. Therefore, to elucidate the biological function of DEMs, we performed miRNA-target interaction and pathway enrichment of predicted targets. All predicted targets of each miRNA were obtained from TargetScan (http:// www.targetscan.org/vert_71/), and only those targets with a cumulative weighted context score less than -0.4 were selected for further analysis. Figure 5 shows the miRNA-target interaction of each candidate miRNA using Cytoscape software (version 3.4.0). The number of predicted targets of a single miRNA ranged from 13 to 169. Seventeen genes (ARHGAP6, BRMS1L, FAM175B, FAM217B, FRS2, KCNJ6, L3MBTL3, MBNL1, MITF, PDCD4, RABGAP1L, RASA2, RGS17, SAMD12, SERP1, SHC4 and TPD52) were targeted by more than one miRNA. We also performed pathway enrichment analysis of the predicted targets. Figure $6 \mathrm{~A}$ shows that
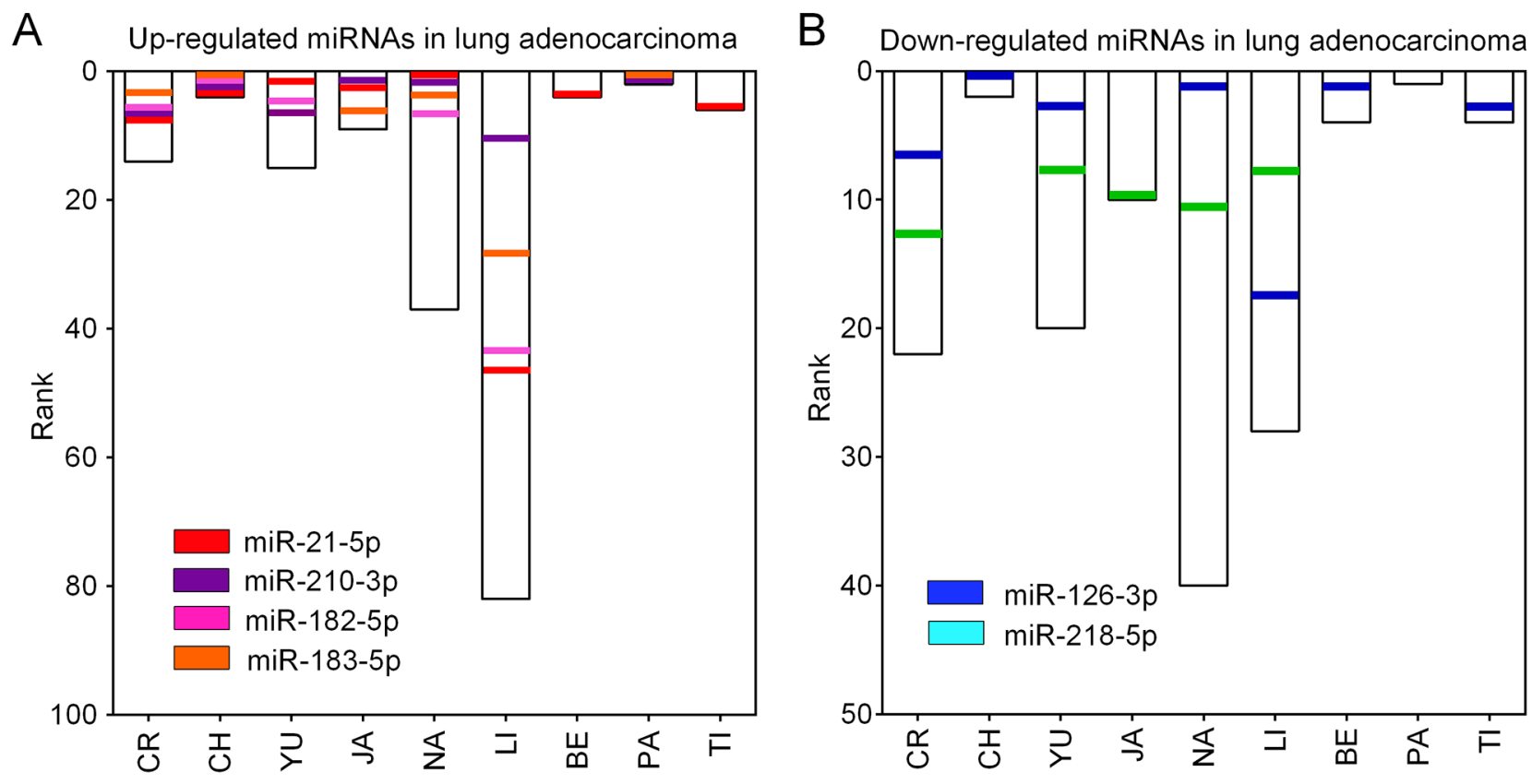

Figure 3: RRA analysis of DEMs in LUAD. (A) Up-regulated and (B) down-regulated miRNAs in LUAD were re-ranked according to their crude and Bonferroni-corrected $P$-values. Only six miRNAs (four up-regulated: miR-21-5p, miR-210-3p, miR-182-5p and miR183-5p; two down-regulated: miR-126-3p and miR-218-5p) reached statistical significance with Bonferroni-corrected $P$-values less than $0.05(-\log 10[P$-value $]>1.30)$. 
five KEGG pathways, including the "MAPK signaling pathway", "Neurotrophin signaling pathway", "Pathway in cancer", "Regulation of actin cytoskeleton" and "Axon guidance pathway", were associated with more than three miRNAs. Another algorithm (DIANA-miRPath v3.0) predicted the pathways affected by multiple miRNAs as "Pathway in cancer", "Regulation of actin cytoskeleton", "MAPK signaling pathway" and "Axon guidance pathway", which were also common pathways affected by the largest number of miRNAs (Table 2). Notably,

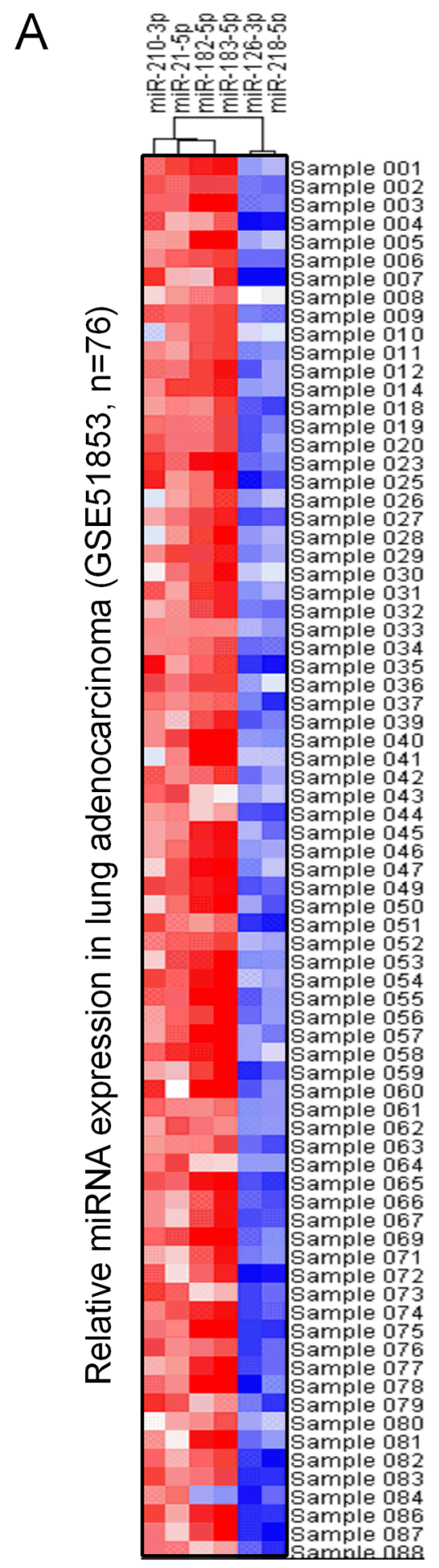

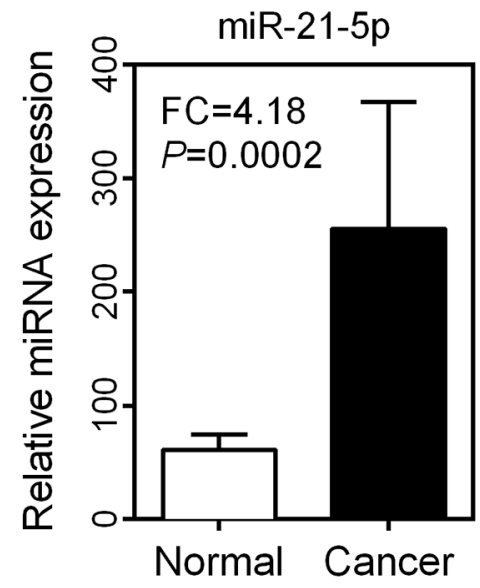
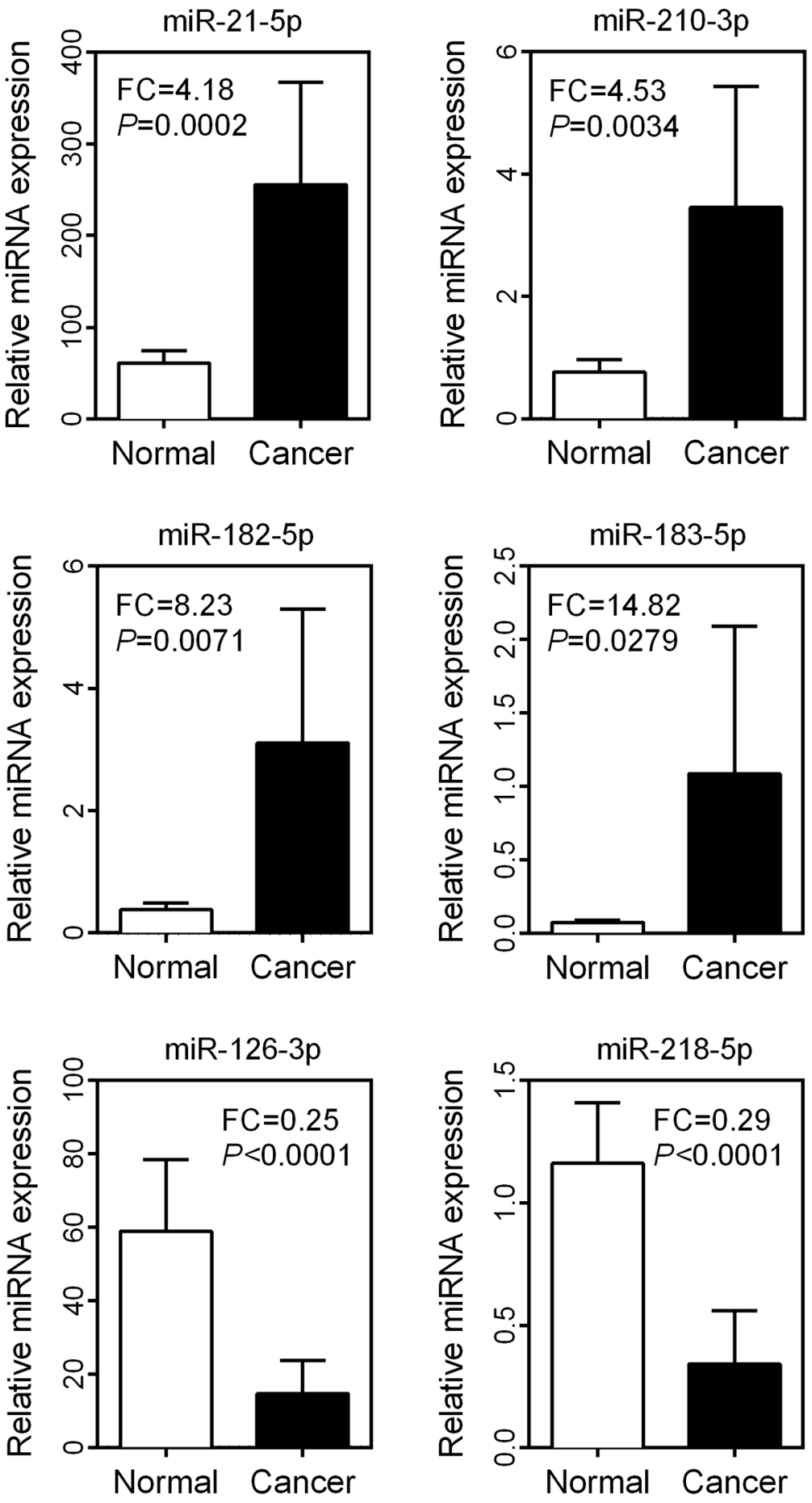

Figure 4: Expression validation of six DEMs in LUAD. (A) Expression levels of six DEMs in 76 LUAD tissue samples were visualized as a heatmap. (B) Comparison of six DEM expression levels between normal and cancer groups. 
we found that the "Estrogen signaling pathway" was the most significant pathway affected by six miRNAs (Figure $6 \mathrm{~B})$. Activation of the estrogen signaling pathway was a reported promoter for LUAD [17], and our findings support critical roles for these six miRNAs in LUAD development.

A

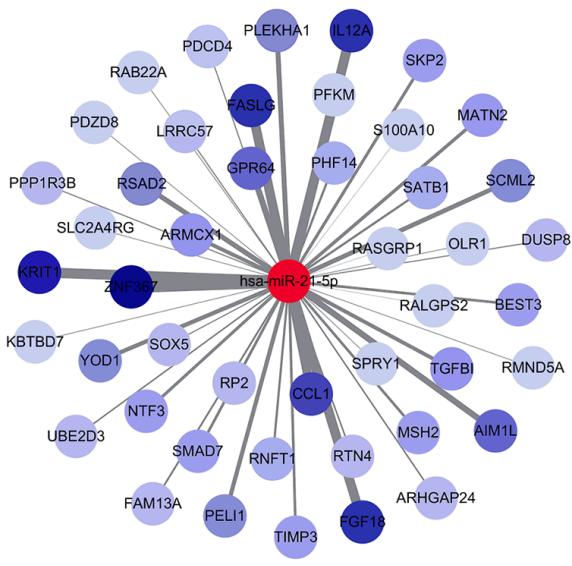

C

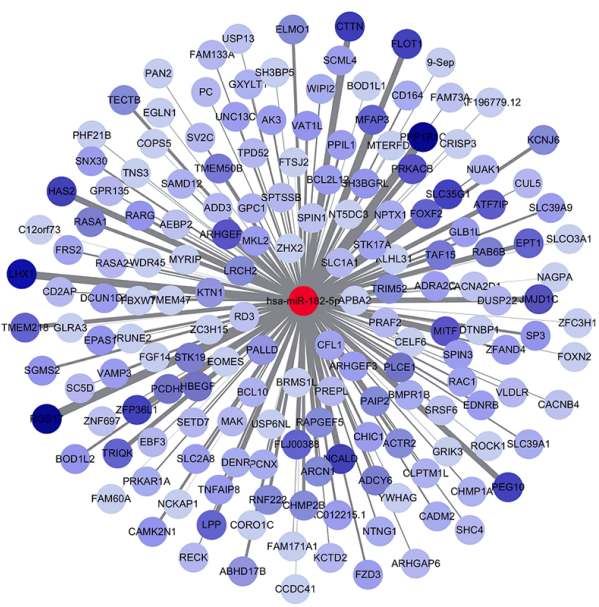

$E$

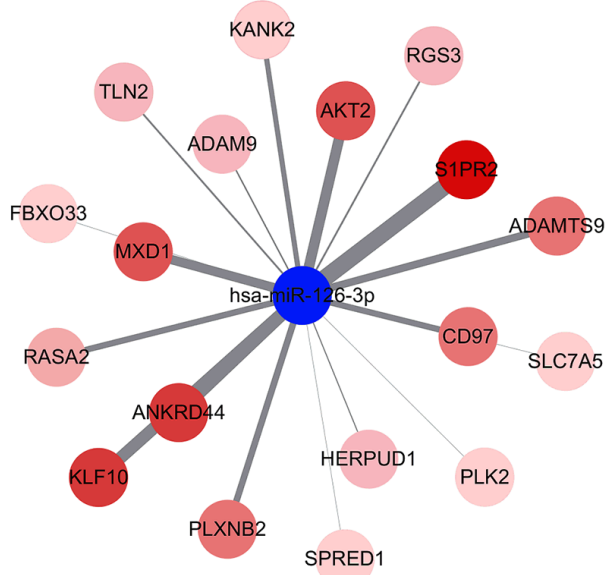

\section{The association between DEMs and clinical} outcome in LUAD

To explore the prognostic values of DEMs in LUAD patients, we obtained the LUAD TCGA dataset from SurvMicro (http://bioinformatica.mty.itesm.mx/
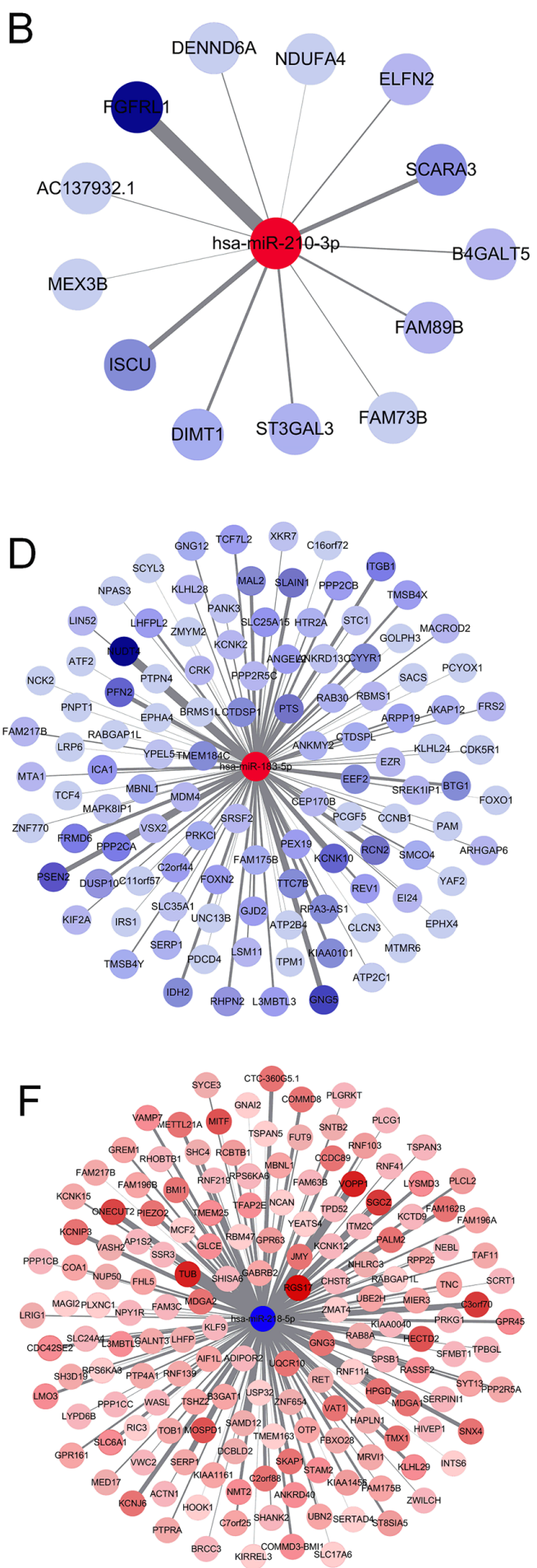

Figure 5: MiRNA-gene interaction network. (A) miR-21-5p, (B) miR-210-3p, (C) miR-182-5p, (D) miR-183-5p, (E) miR-126-3p and (F) miR-218-5p. 
SurvMicro), which is a web-based tool for assessing miRNA-based prognostic signatures [18]. We evaluated the association between miRNAs and patients' overall survival using two panels, including four up-regulated miRNAs (miR-21-5p, miR-210-3p, miR-182-5p and miR-183-5p) and two down-regulated miRNAs (miR126-3p and miR-218-5p). Patients in each panel were divided into two groups, high-risk and low-risk groups, according to the prognostic index calculated by multivariate survival analysis using the SurvMicro web tool. Figure 7A shows that the panel of four up-regulated miRNAs was significantly associated with overall survival of LUAD, with a $P$-value of 0.0035 . Patients in the high-risk group exhibited lower cumulative survival rates than the low-risk group (hazard ratio $[\mathrm{HR}]=2.23$, corresponding $95 \%$ confidence interval $[95 \% \mathrm{CI}]=1.30$ 3.83). Similarly, the panel of two down-regulated miRNAs was also significantly associated with overall survival in patients with LUAD (HR $[95 \% \mathrm{CI}]=2.39$ [1.36-4.20], $P=0.0024)$. These findings support the potential prognostic values of these six DEMs in survival prediction for LUAD patients.

\section{DISCUSSION}

In the present study, we combined nine miRome profiling studies and identified LUAD-specific miRNAs from a total of 595 LUAD and 168 non-cancerous tissue samples using the RRA method. A panel of four upregulated miRNAs (miR-21-5p, miR-210-3p, miR-182-5p and miR-183-5p) and two down-regulated miRNAs (miR126-3p and miR-218-5p) were identified as commonly aberrantly expressed miRNAs in LUAD. Functional analysis revealed that these six miRNAs may be involved in LUAD development via modulation of the estrogen signaling pathway. Our clinical investigation further supports the prognostic value of these six miRNAs in LUAD patients.

In recent years, high-throughput profiling methods have been widely used to identify tumor-specific miRNAs as



Figure 6: Pathway enrichment analysis of predicted targets of DEMs. (A) Heatmap of the KEGG pathway enrichment of the target genes of six DEMs. Rows: pathways; Columns: genes. Range of colors (deep red to white) represented the $-\log 10$ (FDR). (B) The combinatorial effects of six DEMs in KEGG pathways. 
Table 2: The combinatorial effect of six DEMs in KEGG pathways

\begin{tabular}{|c|c|c|c|}
\hline \# & KEGG pathway & \#miRNAs & \#genes \\
\hline 1 & Pathways in cancer & 6 & 68 \\
\hline 2 & PI3K-Akt signaling pathway & 5 & 59 \\
\hline 3 & Regulation of actin cytoskeleton & 5 & 47 \\
\hline 4 & Proteoglycans in cancer & 5 & 41 \\
\hline 5 & AMPK signaling pathway & 5 & 25 \\
\hline 6 & Axon guidance & 5 & 24 \\
\hline 7 & Prostate cancer & 5 & 19 \\
\hline 8 & ECM-receptor interaction & 5 & 15 \\
\hline 9 & Rap1 signaling pathway & 4 & 40 \\
\hline 10 & Ras signaling pathway & 4 & 34 \\
\hline 11 & Dopaminergic synapse & 4 & 29 \\
\hline 12 & Oxytocin signaling pathway & 4 & 29 \\
\hline 13 & Adrenergic signaling in cardiomyocytes & 4 & 28 \\
\hline 14 & Viral carcinogenesis & 4 & 28 \\
\hline 15 & Hippo signaling pathway & 4 & 25 \\
\hline 16 & Neurotrophin signaling pathway & 4 & 25 \\
\hline 17 & Oocyte meiosis & 4 & 24 \\
\hline 18 & Cholinergic synapse & 4 & 24 \\
\hline 19 & Platelet activation & 4 & 24 \\
\hline 20 & Glutamatergic synapse & 4 & 22 \\
\hline 21 & Retrograde endocannabinoid signaling & 4 & 21 \\
\hline 22 & Estrogen signaling pathway & 4 & 20 \\
\hline 23 & Morphine addiction & 4 & 18 \\
\hline 24 & Adherens junction & 4 & 18 \\
\hline 25 & GABAergic synapse & 4 & 17 \\
\hline 26 & Amphetamine addiction & 4 & 16 \\
\hline 27 & Long-term potentiation & 4 & 16 \\
\hline 28 & Bacterial invasion of epithelial cells & 4 & 16 \\
\hline 29 & Vasopressin-regulated water reabsorption & 4 & 15 \\
\hline 30 & Cocaine addiction & 4 & 11 \\
\hline 31 & Circadian entrainment & 3 & 18 \\
\hline 32 & Melanogenesis & 3 & 18 \\
\hline 33 & Phosphatidylinositol signaling system & 3 & 17 \\
\hline 34 & Inositol phosphate metabolism & 3 & 14 \\
\hline 35 & TGF-beta signaling pathway & 3 & 14 \\
\hline 36 & Long-term depression & 3 & 13 \\
\hline 37 & $\begin{array}{l}\text { Endocrine and other factor-regulated calcium } \\
\text { reabsorption }\end{array}$ & 3 & 10 \\
\hline
\end{tabular}


biomarkers for cancer diagnostic, prognostic and therapeutic applications [19, 20]. However, due to the use of different methods and platforms, the conclusions varied among those profiling studies. The RRA method was specifically designed to identify the most commonly overlapping factors and make these studies comparable [5]. An increasing number of studies have attempted to examine tumor specific-miRNAs using the RRA method. Researchers used this method in multiple malignancies, including pancreatic, liver, renal, breast, colon, ovarian, bladder, and nasopharyngeal tract cancer [16, 21-27]. Võsa U et al [6] first identified a panel of 15 aberrantly expressed miRNAs in lung cancer, including seven up-regulated miRNAs (miR-21, miR-210, miR-182, miR-31, miR-200b, miR-205 and miR-183) and eight down-
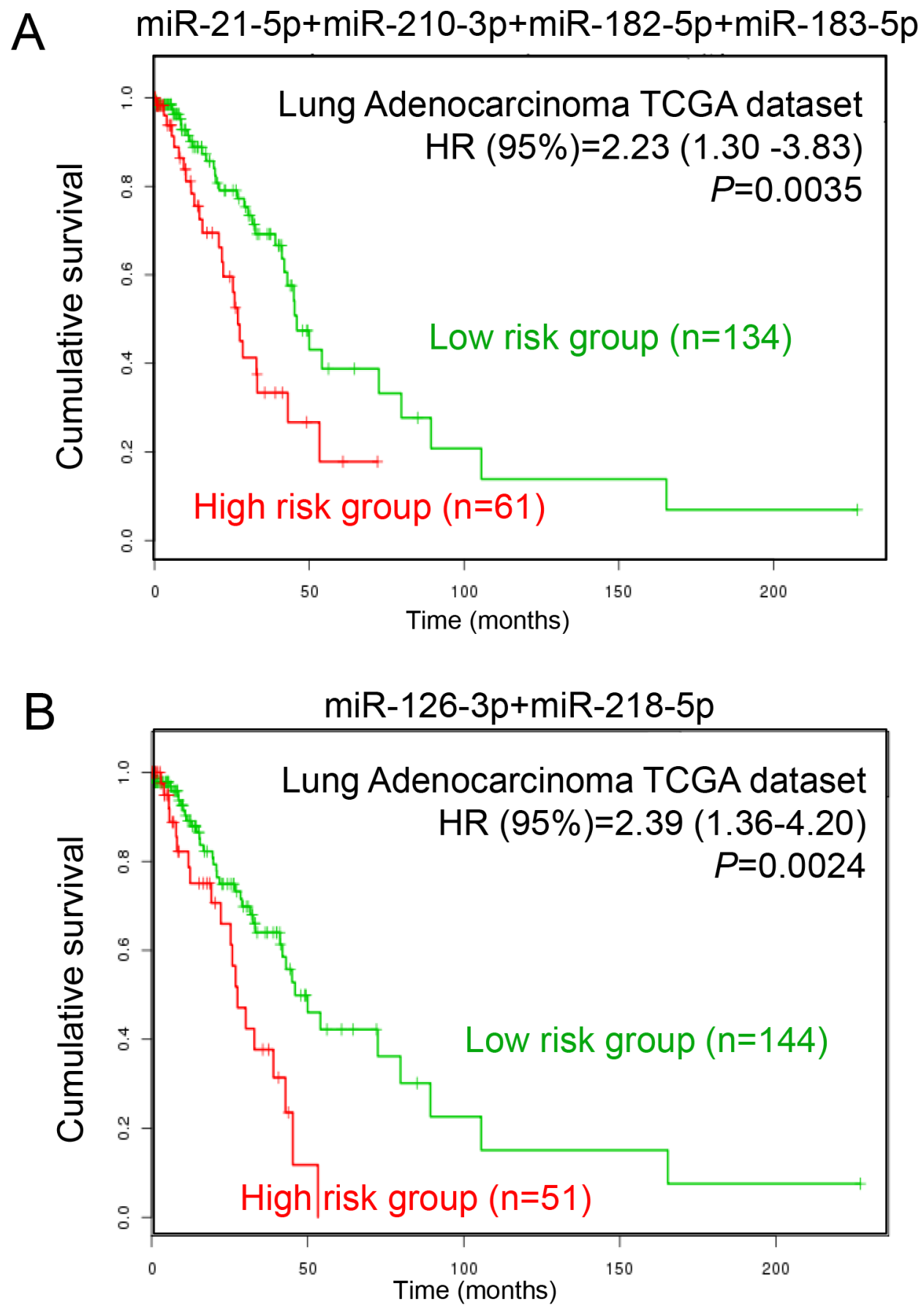

Figure 7: Prognostic analysis of LUAD patients with different miRNA expression levels. (A) The prognostic values of four up-regulated miRNAs (miR-21-5p, miR-210-3p, miR-182-5p and miR-183-5p) in LUAD patients. (B) The prognostic values of two downregulated miRNAs (miR-126-3p and miR-218-5p) in LUAD patients. 
regulated miRNAs (miR-126-3p, miR-30a, miR-30d, miR486-5p, miR-451a, miR-126-5p, miR-143 and miR-145). Their results provided solid evidence for the use of these miRNAs as promising diagnostic and prognostic biomarkers in lung cancer. However, the authors mixed different histological types of lung cancer together and did not analyze miRNA signatures separately. Therefore, their findings based on mixed samples may have limited value in the clinic. Therefore, this study only included miRNome profiling studies of LUAD. Our findings improve our understanding of the molecular mechanism and identify more specific tumor biomarkers for LUAD [28].

Several of the miRNAs identified in our study, such as miR-21-5p and miR-210-3p, are functionally important in the progression of LUAD [29, 30]. When comparing our results with Võsa's results, we identified a specific down-regulated miRNA, miR-218-5p. Previous studies revealed that miR218 acts as a tumor suppressor in many malignancies, such as glioma, head and neck squamous cell carcinoma, and cervical, nasopharyngeal, gastric and colon cancers [31-35]. In non-small cell lung cancer, miR-218 was reported to be significantly down-regulated and affect cell migration and invasion [36, 37]. Patients with low miR-218 expression exhibited a worse prognosis than patients with high miR218 expression [38]. Using LUAD cell lines, Sher YP et al [39] found that the expression of miR-218 was significantly inhibited and involved in the process of brain metastasis. In our study, using an independent cohort, we also found that miR-218 was significantly down-regulated in LUAD tissues (fold change $=0.29 ; P<0.0001$ ). These reports support the need for further experimental and clinical exploration of miR218 and other miRNAs in LUAD.

To further explore the underlying molecular association between DEMs and LUAD, in this study, we also performed miRNA-target interaction network and pathway enrichment analyses. We found that several KEGG pathways, such as "Regulation of actin cytoskeleton" and "MAPK signaling pathway", were affected by nearly all six miRNAs. A very recent study demonstrated that "Regulation of actin cytoskeleton" and "MAPK signaling pathway" contribute to disease progression and drug resistance in LUAD cells [40], which suggests the critical role of DEMs in LUAD. Notably, we also found that the "Estrogen signaling pathway" was the most significant pathway affected by the six miRNAs, with a $P$-value of $4.44 \mathrm{E}-05$. For decades, epidemiological studies have demonstrated an increase in LUAD in women. A mouse model demonstrated that estrogen is an essential factor during LUAD carcinogenesis [17]. Ikeda $\mathrm{K}$ et al [41] observed that the estrogen concentration in LUAD was significantly higher than controls. Moreover, a clinical investigation revealed that postmenopausal women who received estrogen plus progestin treatment exhibit an increased number of deaths from lung cancer [42]. The estrogen signaling pathway was also involved in cellular cytoskeleton organization [43] and cross-talk with the MAPK signaling pathway [44]. Taken together, biological function analyses of these six miRNAs strongly suggest critical roles in LUAD, which may provide a new strategy for LUAD diagnosis and prognosis.

In conclusion, we identified a panel of six miRNAs (miR-21-5p, miR-210-3p, miR-182-5p and miR-183-5p, miR-126-3p and miR-218-5p) in LUAD and evaluated their expression and prognostic values in an independent cohort. Our biological informatics analysis provided evidence for the important roles of these miRNAs in LUAD via modulation of the estrogen signaling pathway. Our findings warrant further clinical investigations to validate these six miRNAs as biomarkers for the early diagnosis and prognosis prediction of LUAD.

\section{MATERIALS AND METHODS}

\section{Selection strategy for miRNome profiling studies}

MiRNome profiling studies that used highthroughput methods, including second-generation sequencing, miRNA microarray and polymerase chain reaction (PCR) methods, which were designed for the parallel quantification of large numbers of miRNAs (96- or 384-well microplates) in LUAD tissue were included. MiRNome profiling studies based on only cell lines and non-LUAD subtypes were excluded. Rank lists of aberrantly expressed miRNAs from each study were mapped according to miRBase Version 21. Non-human miRNA, including viral and other species' miRNAs, were excluded. The miRNA lists were separately recorded as up- or down-regulated miRNAs.

\section{Robust rank aggregation analysis}

RRA analysis was performed using $\mathrm{R}$ software and an R package named "Robust Rank Aggreg" (freely available at comprehensive R Archive Network website, http://cran.r-project.org/). All miRNA lists were re-ranked according to the $P$-value assigned for each miRNA. Bonferroni corrections of $P$-values were calculated to avoid false positive results. Only corrected $P$-values less than 0.05 were considered statistically significant.

\section{MiRNA-gene interaction network analysis}

The TargetScan web tool (www.targetscan.org/) was used to predict the gene target of each DEM. Only gene targets with a cumulative weighted context score $<-0.4$ were selected for inclusion in the miRNA-gene interaction network. The miRNA-gene interaction network was visualized using Cytoscape software (version 3.4.0).

\section{KEGG pathway enrichment analysis}

KEGG pathway enrichment analysis for the predicted gene targets of each DEM was performed using the DAVID web tool (https://david.ncifcrf.gov/). The false discovery rate (FDR) of each KEGG pathway 
was transformed by $-\log 10$ and visualized as a heatmap. The combinatorial effects of multiple miRNAs in KEGG pathways were calculated using the miRPath algorithm in the DIANA web tool (http://www.microrna.gr/ miRPathv2).

\section{Survival analysis}

LUAD patients from The Cancer Genome Atlas (TCGA) cohort were used to investigate the prognosis values of DEMs in LUAD. The prognostic data and Kaplan-Meier curve were calculated on SurvMicro web tools (http://bioinformatica.mty.itesm.mx/SurvMicro).

\section{Statistical analysis}

All data in the present study were analyzed using GraphPad Prism 6.0 software. The expression of each miRNA is expressed as a mean \pm standard error of measurement (SD). Comparisons of each miRNA between cancer and normal groups were performed using Student's $t$-test. A $P$-value less than 0.05 was considered statistically significant.

\section{Author contributions}

Zhuo Peng worked for study search, quality check, data extraction, and analysis as a principal investigator. Longfei Pan, Zequn Niu, Wei Li and Xiaoyan Dang contributed for study conception. Lin Wan and Rui Zhang worked as methodologist. Shuanying Yang critically revised the manuscript and coordinated the study. All authors reviewed and finally approved the manuscript.

\section{CONFLICTS OF INTEREST} of interest.

The authors declare they have no potential conflicts

\section{REFERENCES}

1. Bartel DP. MicroRNAs: genomics, biogenesis, mechanism, and function. Cell. 2004; 116:281-297.

2. Cho WC, Chow AS, Au JS. Restoration of tumour suppressor hsa-miR-145 inhibits cancer cell growth in lung adenocarcinoma patients with epidermal growth factor receptor mutation. Eur J Cancer. 2009; 45:2197-2206.

3. Hayes J, Peruzzi PP, Lawler S. MicroRNAs in cancer: biomarkers, functions and therapy. Trends Mol Med. 2014; 20:460-469.

4. Munker R, Calin GA. MicroRNA profiling in cancer. Clin Sci (Lond). 2011; 121:141-158.

5. Shrestha S, Hsu SD, Huang WY, Huang HY, Chen W, Weng SL, Huang HD. A systematic review of microRNA expression profiling studies in human gastric cancer. Cancer Med. 2014; 3:878-888.
6. Kuosmanen SM, Hartikainen J, Hippelainen M, Kokki H, Levonen AL, Tavi P. MicroRNA profiling of pericardial fluid samples from patients with heart failure. PLoS One. 2015; 10:e119646.

7. Pritchard CC, Cheng HH, Tewari M. MicroRNA profiling: approaches and considerations. Nat Rev Genet. 2012; 13:358-369.

8. Vosa U, Kolde R, Vilo J, Metspalu A, Annilo T. Comprehensive meta-analysis of microRNA expression using a robust rank aggregation approach. Methods Mol Biol. 2014; 1182:361-373.

9. Crawford M, Batte K, Yu L, Wu X, Nuovo GJ, Marsh CB, Otterson GA, Nana-Sinkam SP. MicroRNA 133B targets pro-survival molecules MCL-1 and BCL2L2 in lung cancer. Biochem Biophys Res Commun. 2009; 388:483-489.

10. Yu L, Todd NW, Xing L, Xie Y, Zhang H, Liu Z, Fang H, Zhang J, Katz RL, Jiang F. Early detection of lung adenocarcinoma in sputum by a panel of microRNA markers. Int J Cancer. 2010; 127:2870-2878.

11. Jang JS, Jeon HS, Sun Z, Aubry MC, Tang H, Park CH, Rakhshan F, Schultz DA, Kolbert CP, Lupu R, Park JY, Harris CC, Yang P, Jen J. Increased miR-708 expression in NSCLC and its association with poor survival in lung adenocarcinoma from never smokers. Clin Cancer Res. 2012; 18:3658-3667.

12. Nadal E, Zhong J, Lin J, Reddy RM, Ramnath N, Orringer MB, Chang AC, Beer DG, Chen G. A MicroRNA cluster at 14q32 drives aggressive lung adenocarcinoma. Clin Cancer Res. 2014; 20:3107-3117.

13. Li X, Shi Y, Yin Z, Xue X, Zhou B. An eight-miRNA signature as a potential biomarker for predicting survival in lung adenocarcinoma. J Transl Med. 2014; 12:159.

14. Begum S, Hayashi M, Ogawa T, Jabboure FJ, Brait M, Izumchenko E, Tabak S, Ahrendt SA, Westra WH, Koch W, Sidransky D, Hoque MO. An integrated genome-wide approach to discover deregulated microRNAs in nonsmall cell lung cancer: clinical significance of miR-23b-3p deregulation. Sci Rep. 2015; 5:13236.

15. Pak MG, Lee CH, Lee WJ, Shin DH, Roh MS. Unique microRNAs in lung adenocarcinoma groups according to major TKI sensitive EGFR mutation status. Diagn Pathol. 2015; 10:99.

16. Tian F, Li R, Chen Z, Shen Y, Lu J, Xie X, Ge Q. Differentially expressed miRNAs in tumor, adjacent, and normal tissues of lung adenocarcinoma. BioMed Res Int. 2016; 2016:1428271.

17. Teng Y, Su X, Zhang X, Zhang Y, Li C, Niu W, Liu C, Qu K. miRNA-200a/c as potential biomarker in epithelial ovarian cancer (EOC): evidence based on miRNA metasignature and clinical investigations. Oncotarget. 2016; 7:81621-81633. doi: 10.18632/oncotarget.13154.

18. Hammoud Z, Tan B, Badve S, Bigsby RM. Estrogen promotes tumor progression in a genetically defined mouse model of lung adenocarcinoma. Endocr Relat Cancer. 2008; 15:475-483. 
19. Aguirre-Gamboa R, Trevino V. SurvMicro: assessment of miRNA-based prognostic signatures for cancer clinical outcomes by multivariate survival analysis. Bioinformatics. 2014; 30:1630-1632.

20. Chen X, Shi K, Wang Y, Song M, Zhou W, Tu H, Lin Z. Clinical value of integrated-signature miRNAs in colorectal cancer: miRNA expression profiling analysis and experimental validation. Oncotarget. 2015; 6:37544-37556. doi: 10.18632/oncotarget.6065.

21. Luan J, Wang J, Su Q, Chen X, Jiang G, Xu X. Metaanalysis of the differentially expressed microRNA profiles in nasopharyngeal carcinoma. Oncotarget. 2016; 7:1051310521. doi: 10.18632/oncotarget. 7013 .

22. Shi KQ, Lin Z, Chen XJ, Song M, Wang YQ, Cai YJ, Yang NB, Zheng MH, Dong JZ, Zhang L, Chen YP. Hepatocellular carcinoma associated microRNA expression signature: integrated bioinformatics analysis, experimental validation and clinical significance. Oncotarget. 2015; 6:25093-25108. doi: 10.18632/oncotarget.4437.

23. Tang K, Xu H. Prognostic value of meta-signature miRNAs in renal cell carcinoma: an integrated miRNA expression profiling analysis. Sci Rep. 2015; 5:10272.

24. Wei Y, He R, Wu Y, Gan B, Wu P, Qiu X, Lan A, Chen G, Wang Q, Lin X, Chen Y, Mo Z. Comprehensive investigation of aberrant microRNA profiling in bladder cancer tissues. Tumour Biol. 2016; 37:12555-12569.

25. Yang J, Han S, Huang W, Chen T, Liu Y, Pan S, Li S. A meta-analysis of microRNA expression in liver cancer. PLoS One. 2014; 9:e114533.

26. Zhou H, Tang K, Xiao H, Zeng J, Guan W, Guo X, Xu H, Ye Z. A panel of eight-miRNA signature as a potential biomarker for predicting survival in bladder cancer. J Exp Clin Cancer Res. 2015; 34:53.

27. Vosa U, Vooder T, Kolde R, Vilo J, Metspalu A, Annilo T. Meta-analysis of microRNA expression in lung cancer. Int J Cancer. 2013; 132:2884-2893.

28. Cheng TY, Cramb SM, Baade PD, Youlden DR, Nwogu C, Reid ME. The International Epidemiology of Lung Cancer: latest trends, disparities, and tumor characteristics. J Thorac Oncol. 2016; 11:1653-1671.

29. Li B, Ren S, Li X, Wang Y, Garfield D, Zhou S, Chen X, Su C, Chen M, Kuang P, Gao G, He Y, Fan L, et al. MiR21 overexpression is associated with acquired resistance of EGFR-TKI in non-small cell lung cancer. Lung Cancer. 2014; 83:146-153.

30. Puissegur MP, Mazure NM, Bertero T, Pradelli L, Grosso S, Robbe-Sermesant K, Maurin T, Lebrigand K, Cardinaud B, Hofman V, Fourre S, Magnone V, Ricci JE, et al. miR-210 is overexpressed in late stages of lung cancer and mediates mitochondrial alterations associated with modulation of HIF-1 activity. Cell Death Differ. 2011; 18:465-478.

31. Tu Y, Gao X, Li G, Fu H, Cui D, Liu H, Jin W, Zhang Y. MicroRNA-218 inhibits glioma invasion, migration, proliferation, and cancer stem-like cell self-renewal by targeting the polycomb group gene Bmi1. Cancer Res. 2013; 73:6046-6055.

32. Kinoshita T, Hanazawa T, Nohata N, Kikkawa N, Enokida H, Yoshino H, Yamasaki T, Hidaka H, Nakagawa M, Okamoto Y, Seki N. Tumor suppressive microRNA-218 inhibits cancer cell migration and invasion through targeting laminin-332 in head and neck squamous cell carcinoma. Oncotarget. 2012; 3:1386-1400. doi: 10.18632/ oncotarget.709.

33. Alajez NM, Lenarduzzi M, Ito E, Hui AB, Shi W, Bruce J, Yue S, Huang SH, Xu W, Waldron J, O'Sullivan B, Liu FF. MiR-218 suppresses nasopharyngeal cancer progression through downregulation of survivin and the SLIT2-ROBO1 pathway. Cancer Res. 2011; 71:2381-2391.

34. Tie J, Pan Y, Zhao L, Wu K, Liu J, Sun S, Guo X, Wang B, Gang Y, Zhang Y, Li Q, Qiao T, Zhao Q, et al. MiR218 inhibits invasion and metastasis of gastric cancer by targeting the Robol receptor. PLoS Genet. 2010; 6:e1000879.

35. He X, Dong Y, Wu CW, Zhao Z, Ng SS, Chan FK, Sung JJ, Yu J. MicroRNA-218 inhibits cell cycle progression and promotes apoptosis in colon cancer by downregulating BMI1 polycomb ring finger oncogene. Mol Med. 2013; 18:1491-1498.

36. Zhang C, Ge S, Hu C, Yang N, Zhang J. MiRNA-218, a new regulator of HMGB1, suppresses cell migration and invasion in non-small cell lung cancer. Acta Biochim Biophys Sin. 2013; 45:1055-1061.

37. Zhu K, Ding H, Wang W, Liao Z, Fu Z, Hong Y, Zhou Y, Zhang CY, Chen X. Tumor-suppressive miR-218-5p inhibits cancer cell proliferation and migration via EGFR in non-small cell lung cancer. Oncotarget. 2016; 7:2807528085. doi: 10.18632/oncotarget.8576.

38. Wu DW, Cheng YW, Wang J, Chen CY, Lee H. Paxillin predicts survival and relapse in non-small cell lung cancer by microRNA-218 targeting. Cancer Res. 2010; 70:10392-10401.

39. Sher YP, Wang LJ, Chuang LL, Tsai MH, Kuo TT, Huang CC, Chuang EY, Lai LC. ADAM9 up-regulates N-cadherin via miR-218 suppression in lung adenocarcinoma cells. PLoS One. 2014; 9:e94065.

40. Fang $\mathrm{Y}$, Zhang $\mathrm{C}$, Wu $\mathrm{T}$, Wang Q, Liu J, Dai $\mathrm{P}$. Transcriptome sequencing reveals key pathways and genes associated with cisplatin resistance in lung adenocarcinoma A549 cells. PLoS One. 2017; 12:e0170609.

41. Ikeda K, Shiraishi K, Yoshida A, Shinchi Y, Sanada M, Motooka Y, Fujino K, Mori T, Suzuki M. Synchronous multiple lung adenocarcinomas: estrogen concentration in peripheral lung. PLoS One. 2016; 11:e0160910.

42. Chlebowski RT, Schwartz AG, Wakelee H, Anderson GL, Stefanick ML, Manson JE, Rodabough RJ, Chien JW, Wactawski-Wende J, Gass M, Kotchen JM, Johnson $\mathrm{KC}$, O’Sullivan MJ, et al. Oestrogen plus progestin and lung cancer in postmenopausal women (Women's Health 
Initiative trial): a post-hoc analysis of a randomised controlled trial. Lancet. 2009; 374:1243-1251.

43. Moy I, Todorovic V, Dubash AD, Coon JS, Parker JB, Buranapramest M, Huang CC, Zhao H, Green KJ, Bulun SE. Estrogen-dependent sushi domain containing 3 regulates cytoskeleton organization and migration in breast cancer cells. Oncogene. 2015; 34:323-333.
44. Huff MO, Todd SL, Smith AL, Elpers JT, Smith AP, Murphy RD, Bleser-Shartzer AS, Hoerter JE, Radde BN, Klinge CM. Arsenite and cadmium activate MAPK/ERK via membrane estrogen receptors and G-protein coupled estrogen receptor signaling in human lung adenocarcinoma cells. Toxicol Sci. 2016; 152:62-71. 